\title{
DiOTtols
}

Revista de Comunicación Digital

\section{Los emojis del 8M: Su uso en Twitter durante las movilizaciones feministas de 2019}

\section{The 8M emojis: Their use on Twitter during the 2019 feminist mobilizations}

Agnese Sampietro

sampietr@uji.es

Universitat Jaume I

\section{Dafne Calvo}

dafne.calvo@uva.es

Universidad de Valladolid

\section{Eva Campos-Domínguez}

eva.campos@hmca.uva.es

Universidad de Valladolid

Este trabajo ha sido realizado en el marco del proyecto nacional de I+D+i Estrategias, agendas y discursos en las cibercampañas electorales: medios de comunicación y ciudadanos (CSO2016-77331C2-1-R), dirigido por Guillermo López García y financiado por el Ministerio de Economía y Competitividad para el período 2017-2020.

Recibido: 05/12/2019 Aceptado: 06/03/2020

\section{Resumen}

Los emojis se han convertido en un elemento prototípico de la comunicación digital y han atraído considerable atención académica en los últimos años, pero su uso coincidiendo con eventos específicos no ha sido analizado sistemáticamente. El presente trabajo explora el uso de los emojis en Twitter en ocasión de las manifestaciones y huelgas convocadas en España el 8 de marzo (8M) de 2019, Día Internacional de la Mujer, analizando un corpus de más de medio millón de tuits publicados del 4 al 9 de marzo de 2019.

Los resultados muestran que el uso que se hace en Twitter de estos pictogramas alrededor del $8 \mathrm{M}$ es bastante diferente del uso general en la red social. Además de un mínimo uso de las caritas amarillas (como ) que suelen predominar en Twitter, el emoji más utilizado en el corpus es el corazón de color violeta $(\vartheta)$, que se está convirtiendo en símbolo del ciberactivismo feminista en España. Son muy frecuentes también los emojis que representan gestos: algunos, como el puño en alto se relacionan con la iconografía de la protesta; otros, como el dedo índice, son simplemente gestos deícticos que enfatizan visualmente enlaces o adjuntos. Se vislumbran incluso patrones en el uso de los emojis que dependen de la ideología política. En general, este trabajo muestra que los emojis se usan de forma altamente simbólica en situaciones de relevancia social, convirtiéndose en un instrumento para el discurso feminista en internet.

\section{Palabras clave}

emojis, Twitter, feminismo, ciberactivismo, 8M

Cómo citar este artículo:

Sampietro, A.; Calvo, D. y Campos-Domínguez, E. (2020). Los emojis del 8M: Su uso en Twitter durante las movilizaciones feministas de 2019. Dígitos. Revista de Comunicación Digital, 6: 137-158. DOI: 10.7203/ rd.v1i6.170 


\begin{abstract}
Emojis have become a typical feature of digital communication and have attracted considerable academic attention in recent years. Nonetheless, their use on occasion of specific events has not been systematically analyzed. This paper explores the use of emojis on Twitter during demonstrations and strikes held in Spain on March 8th, 2019, International Women's Day, analyzing a corpus of more than half a million tweets published from 4th to 9th March 2019.

Results show that the use of these pictograms on Twitter around that date is quite different from the general use in the social network. The recognized yellow faces (like ) that usually predominate on Twitter, are way less used in the corpus; by contrast, the most used emoji is the purple heart $(\nabla)$, which is becoming a symbol of feminist cyber-activism in Spain. The emojis that represent gestures are also very frequent: some of them, like the raised fist, are related to the iconography of the protest; others, such as the pointing index finger, are simply deictic gestures that visually emphasize links or attachments. Emerging patterns in the use of emojis that depend on political ideology can also be observed. In general, this work shows that emojis are used in a highly symbolic way in situations of social relevance, becoming an instrument for feminist discourse on the internet.
\end{abstract}

\title{
Keywords
}

emojis, Twitter, feminism, cyber-activism, 8th March

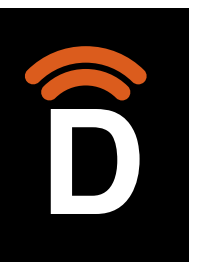

Los emojis del 8M:

Su uso en Twitter durante las movilizaciones feministas de 2019

\section{Introducción}

La conmemoración del 8 de marzo (8M), Día Internacional de la Mujer, congrega en todo el mundo a millones de mujeres de diferente procedencia y estatus social para reivindicar mejoras sociales, laborales y familiares, entre otras. Institucionalizado por Naciones Unidas en 1975, el día rememora un trágico accidente acaecido en una fábrica textil de Nueva York en 1908, donde un incendio provocó la muerte de cientos de obreras que trabajaban en la fábrica (International Women's Day, n.d.). Unos años después, fue propuesto ese día para celebrar el Día Internacional de la Mujer, que nacía con el objetivo de pedir mejoras en los derechos de las mujeres y favorecer la igualdad entre sexos (Ruiz Franco, 2018).

Desde hace unos años, e inspiradas por la Marcha Estatal Contra las Violencias Machistas del 7 de noviembre de 2015, las celebraciones de este evento en España han sido muy notorias, sobre todo por la alta participación (Campillo, 2019; Núñez y Fernández Romero, 2017). Además, desde 2018 varias organizaciones feministas, partidos, sindicatos y ONG convocan ese día una huelga laboral, de cuidados y de consumo, además de manifestaciones multitudinarias en varias ciudades (Comisión 8M, 2019). Las Delegaciones del Gobierno estimaron que la afluencia a la manifestación del $8 \mathrm{M}$ de 2019 fue de cerca de 350.000 personas en Madrid, 230.000 en Barcelona y 220.000 en Valencia (Gómez, 2019).

Ante tan amplia participación, y teniendo en cuenta el panorama actual, no es de 
extrañar que este evento haya tenido mucha repercusión incluso en las redes. Baste con mencionar que el 8 de marzo de 2019, 16 de las 20 principales tendencias (es decir, las palabras claves y etiquetas más destacadas en un determinado momento) en la red social Twitter en España estuvieron relacionadas con la efeméride ("Trending topics del viernes 08 de marzo en España", n.d.)'. Las etiquetas se han convertido, de hecho, en una estrategia empleada por los colectivos feministas a nivel internacional para la difusión de lemas que buscan la relevancia en la agenda política y la gestión de un discurso contrahegemónico en cuestiones de género (Chenou y Cepeda-Másmela, 2019; Eslen-Ziya, 2013; Pavan y Mainardi, 2018).

Este trabajo se basa en un corpus de tuits recogidos coincidiendo con las manifestaciones y los paros del 8M 2019. La observación de la actividad de esos días en Twitter muestra una gran variedad de imágenes (fotos de las manifestaciones, reproducciones de pancartas), vídeos (del evento, promocionales), eslóganes y noticias. Un aspecto que Ilama la atención, además, es el gran despliegue de emojis, como, por ejemplo, el corazón violeta $(\vartheta$ ), que acompañaban a las publicaciones en esa red social relacionadas con el $8 \mathrm{M}$. En este trabajo nos proponemos analizar precisamente el uso que se hizo de los emojis en Twitter en este corpus de tuits relacionados con el 8M 2019, para explorar su valor como instrumento de ciberactivismo. La investigación se estructura como sigue: en el marco teórico se recorrerá la historia e investigación académica sobre los emojis, además de nociones relacionadas con el ciberactivismo. Después se describirá el corpus de tuits recogido y el procedimiento de análisis y se pasará a la presentación de los resultados. Cierran el trabajo la discusión de los resultados y las conclusiones.

\section{Marco teórico}

\subsection{Los emojis}

Los emojis son pequeños pictogramas en color (como $*$ ), que se añaden a los mensajes electrónicos en diferentes plataformas digitales. Se consideran una versión algo más moderna de los emoticonos y los kaomoji, secuencias de signos de puntuación que representan esquemáticamente expresiones faciales ${ }^{2}$. Los emojis fueron diseñados por primera vez en Japón a principios de los años 90 (Burge, 2019) y tuvieron un gran éxito en ese país, al punto que en poco tiempo los principales operadores telefónicos introdujeron sus propios conjuntos de pictogramas. Para solucionar los problemas de compatibilidad entre diferentes empresas de telefonía, los emojis fueron incluidos en el estándar Unicode en 2009.

Su difusión internacional empezó desde que Apple integró un teclado de emojis en su sistema operativo iOS en 2011 y Android hizo lo mismo dos años después. A partir de ese momento los emojis comenzaron a tener un enorme éxito entre los usuarios.

1. Twitter, creada en 2006, es una popular red social (según Statista, 2020, en 2019 tenía más de 275 millones de usuarios) en la que los usuarios publican e interactúan por medio de mensajes breves (máximo 280 caracteres) conocidos como "tuits". Debido a estas limitaciones de espacio, los usuarios tienen a disposición otras affordances (Hutchby, 2001): además de imágenes y vídeos, se pueden añadir enlaces, menciones directas a otros usuarios, etiquetar las publicaciones e incluir emojis, GIFs y coordenadas geográficas.

2. Los emoticonos, como:), fueron creados en Estados Unidos en 1982 y tuvieron difusión en Occidente. Los kaomoji, como ( $ح$ ), también difundidos a partir de los años 80, al contrario, se utilizaron preferentemente en Japón y otros países orientales, pues en su diseño se hace referencia a la iconografía del manga, del anime $y$, como en esos géneros, el énfasis emocional de la expresión son los ojos. A diferencia de los emoticonos, los kaomoji se leen horizontalmente y suelen ser más elaborados (Tang Khe Foon Hew, 2019). 
Por ejemplo, en 2017 se enviaron diariamente más de 5.000 millones de emojis solo en la plataforma Facebook Messenger (Burge, 2017) y se calcula que más de la mitad de los posts de Instagram contienen al menos un emoji (Kmieckowiak, 2017). Es más, las peticiones populares para añadir nuevos emojis al estándar Unicode no paran de crecer, al punto que la actualización número 12 del estándar (correspondiente a 2019) contenía nada menos que 3.017 emojis.

El uso creciente de los emojis por parte de los usuarios de los dispositivos digitales ha despertado en los últimos años el interés académico hacia el uso de estos pictogramas. Los emojis han sido estudiados desde varios ámbitos disciplinarios, como la psicología (Riordan, 2017), el marketing (Ge y Gretzel, 2018; Casado-Molina et al., 2019), los estudios de mercado (Jaeger, Cardello y Schutz, 2013; Jaeger, Roigard y Ares, 2018), el procesamiento del lenguaje natural (Barbieri, Espinosa-Anke y Saggion, 2016; Barbieri, Ballesteros y Saggion, 2017), la semiótica (Danesi, 2016) y la lingüística (Herring y Dainas, 2017; Al Rashdi, 2018; König, 2019; Sampietro, 2019).

Aunque se considere que los emojis, como sus antecedentes tipográficos, los emoticonos, se utilizan para expresar emociones en el ámbito digital (Riordan, 2017; Kabir y Marlow, 2019), estudios recientes han identificado que estos pictogramas llevan a cabo principalmente una función clarificadora del texto escrito que acompañan (Herring y Dainas, 2017). Este resultado contrasta con el prejuicio, bastante difundido, de que los emojis puedan ser una especie de nuevo lenguaje visual de alcance universal (Miyake, 2007; Azuma y Ebner, 2008; Danesi, 2016).

Si bien los estudios lingüísticos sobre los emojis aportan datos interesantes con respecto a su uso, una de las limitaciones de estos trabajos es que analizan únicamente contextos de tipo conversacional, como los mensajes de WhatsApp (Al Rashdi, 2018; Cantamutto y Vela Delfa, 2019; Pérez-Sabater, 2019; Sampietro, 2019) o los hilos de Facebook (Herring y Dainas, 2017; Matamoros-Fernández, 2018). Por el contrario, el uso y las funciones de los emojis en contextos no conversacionales ha sido poco explorado. Entre los trabajos de este tipo destaca el estudio de Ge y Herring (2018) sobre las secuencias de emojis empleadas por personajes célebres en la plataforma china Sina Weibo. Otro famoso del que se ha estudiado el uso de estos pictogramas es Cher. La cantante americana incluye habitualmente el emoji que representa un inodoro en los tuits referidos al presidente Donald Trump, lo que se ha considerado una manera de crear cercanía y afiliación con sus seguidores (Parkwell, 2019). Por otra parte, también se ha analizado el uso de los emojis como estrategia de marketing por parte de las marcas (Ge y Gretzel, 2018; Casado-Molina et al., 2019), Ilegando a la conclusión que estos son muy eficaces para aumentar la implicación de los seguidores.

El uso de los emojis por parte de los ciudadanos de a pie coincidiendo con eventos señalados ha tenido un escaso interés a nivel académico. Hasta el momento los análisis de Hamdan Azhar sobre los emojis más utilizados en Twitter en determinadas fechas, publicados en varios blogs, son las únicas aproximaciones sistemáticas a este fenómeno. Por ejemplo, este autor analizó los 10 emojis más utilizados en Twitter junto con las etiquetas más populares relacionados con la votación para el Brexit (Azhar, 2016a). Aunque el emoji más frecuente seguía siendo el popular face with tears of joy (uno de los más usados en Twitter), el autor observó que el segundo emoji de la lista era la bandera del Reino Unido, lo que indica que en ese corpus dicha bandera era 400 veces más probable de ser utilizada que en el uso general. Aunque ese emoji se encontraba principalmente en los tuits que incluían la etiqueta \#voteleave (es decir, a favor de la salida del Reino Unido de la Unión Europea), un breve análisis cualitativo del 
corpus mostró que la bandera británica se utilizaba no solo de manera nacionalista, sino también de forma decorativa o como manera abreviada de aludir al país en cuestión (Azhar, 2016a).

En otra ocasión, el mismo autor (Azhar, 2017b) examinó brevemente cuáles eran los 5 emojis más utilizados en enero de 2017junto a determinados hashtags correspondientes con varios eventos de importancia en Estados Unidos: \#NoBanNoWall (referente a la prohibición de entrar en Estados Unidos para personas procedentes de países de mayoría musulmana), \#NotMyPresident (que usan los detractores de Trump para distanciarse de sus políticas o declaraciones), \#TheResistance (un movimiento político que protesta contra el presidente Trump) y \#WomensMarch (el hashtag oficial de la marcha de las mujeres en Washington que tuvo lugar el 21 de enero de 2017). Estos eventos tenían en común varios emojis: la bandera americana, el corazón rojo y el emoji raised fist, que representa un puño levantado hacia arriba ; is ; en todos los temas, excepto \#NoBanNoWall también se encontraba entre los más frecuentes la carita face with tears of joy (@). Esta carita sonriente y el corazón rojo son, según Emojitracker (una web que muestra el uso de los emojis en Twitter en tiempo real), dos de los emojis más utilizados en términos absolutos en esa red social. Azhar (2017b) se sorprendió por la prevalencia del puño en alto. En su opinión, este emoji se está convirtiendo en un símbolo de las protestas progresistas y en un signo de solidaridad. Este gesto, en efecto, se encontró también en otro corpus analizado por el mismo autor, el del movimiento \#MeToo (Azhar, 2017a): entre los emojis más empleados se encontraban varios corazones (el rojo, el partido, el violeta, dos corazones), caritas tristes o enfadadas, un dedo indicando hacia abajo, y gestos de fuerza y resistencia como el puño en alto y el bíceps.

\subsection{Ciberactivismo y hashtags en Twitter}

Como se ha indicado en el apartado precedente, si el uso de los emojis en Twitter en determinados momentos clave a nivel socio-político no ha sido objeto de reflexión académica, no es así en el caso de otro fenómeno típico de esa red social: el uso de etiquetas específicas en determinadas ocasiones. Una etiqueta (en inglés hashtag) es una palabra clave que identifica un tema y en la que se puede hacer clic para acceder a otros contenidos con la misma etiqueta ("Etiqueta (internet)", 2019). Generalmente, estos hipervínculos van precedidos por una almohadilla (\#). Los hashtags, creados en 2007, empezaron a utilizarse en Twitter en torno al 2009, pero actualmente varias redes sociales, como Telegram, Facebook, Instagram, etc., también integran estas etiquetas de metadatos.

El uso y popularizaciones de etiquetas específicas en situaciones de protesta no es algo nuevo. El que se suele recordar como el primer evento en el que se crearon hashtags específicos fueron las protestas post-electorales en Irán de 2009 ("Etiqueta (internet)", 2019). Este uso se popularizó tanto que, en ocasión de las movilizaciones del movimiento Occupy Wall Street, la prensa incluso ideó un término específico, hashtag activism, es decir, activismo mediante etiquetas (Augenbraun, 2011), una forma de protesta virtual guiada por hashtags específicos.

El activismo mediante etiquetas ha sido frecuentemente empleado en el movimiento feminista para articular discursos en redes sociales que visibilicen sus reivindicaciones y subviertan los discursos institucionales sobre la cuestión del género: \#NiUnaMenos acompañó a las protestas argentinas contra los feminicidios (Chenou y CepedaMásmela, 2019); \#WhylStayed criticaba la culpabilización de la víctima a nivel mediático 
en los casos de violencia de género (Clark, 2016), \#MeToo generaba solidaridad entre las víctimas de violencia machista (Orgad \& Gill, 2019) y \#MasculinitySoFragile apuntaba a los comportamientos machistas en las redes sociales (Banet-Weiser y Miltner, 2016). Algunos de estos hashtags, como \#NiUnaMenos y \#VivasNosQueremos, que todavía se usan, se convirtieron en poderosos símbolos del activismo feminista a nivel internacional (Núñez Puente, D’Antonio Maceiras y Fernández Romero, 2019).

Con respecto al evento que nos interesa, el 8M, Núñez Puente et al. (2019) analizaron los tuits con las etiquetas \#8M y \#NiUnaMenos durante las protestas del $8 \mathrm{M}$ en 2017 y ratificaron la capacidad del movimiento feminista para instrumentar las redes sociales para un uso político. La investigación de las huelgas de 2018 y 2019 aún es incipiente, si bien los trabajos publicados coinciden en afirmar que las redes sociales contribuyeron a canalizar la organización de las protestas y visibilizar el significado en todos los escenarios de la vida sociales que plantea este nuevo ciclo de protestas feministas (Fernández Romero y Sánchez Duarte, 2019; Galdón, 2018).

Como hemos señalado, el análisis el uso de los emojis en momentos políticos y sociales de relevancia es un tema académico en gran medida inexplorado. En consecuencia, el objetivo principal de este trabajo es el de investigar el uso de los emojis en Twitter coincidiendo con otro evento relevante en España, las exitosas manifestaciones organizadas el $8 \mathrm{M}$ de 2019. Las preguntas de investigación que guían este trabajo son las siguientes:

Pregunta 1. ¿Cuáles son los emojis más utilizados en los tuits sobre el $8 \mathrm{M}$ de 2019 ?

Pregunta 2. ¿En qué se diferencia el uso de los emojis en el corpus analizado del uso de los emojis en Twitter en general?

Pregunta 3. ¿Cuáles son los principales patrones de uso de los emojis más frecuentes en las manifestaciones feministas del 8M?

\title{
3. Método
}

\subsection{Corpus}

El corpus de tuits analizado en este trabajo fue recogido a través de la herramienta Brandwatch Analytics ("Brandwatch", 2019), y se recopilaron todas las conversaciones publicadas en Twitter, en el marco geográfico de España, asociadas a las siguientes etiquetas desde el día 4 al 9 de marzo de 2019:

\begin{abstract}
\#HuelgaFeminista, \#HuelgaFeminista2019, \#HuelgaFeminista8M, \#8M, \#8M2019, \#8deMarzo, \#HaciaLaHuelgaFeminista, \#HaciaLaHuelgaFeminista2019, \#EnHuelga, \#YoParo, \#NosotrasParamos, \#YoVoy8M, \#HuelgadeConsumo, \#HuelgaDeCuidados, \#HuelgaLaboral, \#HuelgaEstudiantil, \#HuelgaEstudiantil8M, \#HuelgaDeConsumo8M, \#HuelgaDeCuidados8M, \#HuelgaLaboral8M, \#1000Motivos, \#NiUnPasoAtras, \#8DiasDeRevuelta
\end{abstract}

Brandwatch Analytics es una herramienta de "escucha" de redes sociales (social listening), capaz de rastrear foros, vídeos, reseñas y redes sociales, como Twitter o Facebook. Dirigida sobre todo a profesionales del ámbito del marketing, permite monitorear un determinado tema o marca, también en directo, mediante búsquedas por hashtags específicos, perfiles de Twitter, operadores por redes sociales, etc. ("Brandwatch", 2019). Una de las desventajas de la herramienta es que no permite guardar y exportar en su 
totalidad los datos recogidos, que solo se pueden acceder a través de la plataforma. Esta, por su parte, se limita a capturar los enlaces a las publicaciones (en este caso, los tuits). En consecuencia, algunos de los tuits recogidos durante las fechas señaladas se perdieron, o bien porque fueron borrados por el usuario, o bien porque un perfil pasó de público a privado. A fecha de finales de julio de 2019, el corpus estaba compuesto de 529.738 tweets (incluyendo los retuits).

\subsection{Procedimiento}

Como hemos mencionado anteriormente, faltan trabajos académicos específicos sobre los emojis que trabajen con corpus similares al de esta investigación. Por un lado, la mayoría de los estudios anteriores sobre los emojis se centran en el uso de estos pictogramas en contextos conversacionales (ver apartado 2.1). Por otro lado, en general, los trabajos sobre el uso de los emojis en la red social Twitter utilizan técnicas de procesamiento del lenguaje natural sobre en corpus de gran escala, analizando la valencia emocional de tuits con emojis (Soranaka y Matsushita, 2012), el grado de similitud entre emojis diferentes (Wijeratne et al., 2017) o las diferencias en el uso de emojis según la lengua o la procedencia geográfica (Barbieri et al., 2016), entre otros temas.

Asimismo, no hemos encontrado trabajos académicos sobre el uso de los emojis durante eventos específicos, como en este caso el 8M (pero véase Cantamutto y Vela Delfa, este volumen). Como los únicos antecedentes, descritos en el marco teórico, son los análisis de Azhar (2016a, 2016b, 2017b, 2017a), publicados en varios blogs, se adopta una metodología similar a la que se describe en esos trabajos: se ha considerado el uso de los emojis más frecuentes en un corpus que contiene tuits relacionados con un evento específico, las protestas del 8M. Sin embargo, a diferencia de Azhar, se incluye un mayor número de emojis, que se ha categorizado y comparado someramente con el uso general que se hace en Twitter y no se han estudiado solo las palabras claves, sino que se ha analizado discursivamente los tuits con los emojis más frecuentes en su conjunto.

Para el análisis de los emojis se ha hecho uso de las posibilidades que ofrecía la herramienta Brandwatch. En primer lugar, se ha extraído de forma automática la lista de los 300 emojis más utilizados y su distribución a lo largo de los días estudiados. En un segundo momento, estos 300 emojis han sido catalogados a partir de las categorías empleadas oficialmente tanto en Emojipedia ${ }^{3}$ como en los principales sistemas operativos y programas que integran teclados de emojis, en los que los pictogramas están repartidos en diferentes pestañas (a las que a veces se añade una sección con los últimos emojis utilizados). Las categorías/pestañas son las siguientes: 1) caras, personas y gestos; 2 ) animales y naturaleza; 3 ) comida y bebida; 4) actividades; 5) viajes y lugares; 6) objetos; 7) símbolos; 8) banderas. Para evitar atribuciones subjetivas a estas categorías, se ha comprobado en qué pestaña se situaba cada emoji en el catálogo oficial de Unicode. Por ejemplo, las reconocidas caritas amarillas se colocan en la Categoría 1 ("caras, personas y gestos"), pero en esa sección se incluyen incluso algunos complementos de vestuario, que se podrían también considerar objetos, como un anillo, zapatos o bolsos. Como estos complementos en el catálogo oficial se incluyen en la categoría de "caras, personas y gestos", hemos mantenido la misma clasificación. Esta categorización del corpus de emojis ha sido útil para poder identificar tendencias en el uso de los 300 emojis más frecuentes, pues ese elevado número de pictogramas 
se ha reducido a 8 categorías. Esta lista de las categorías de emojis más utilizados se ha comparado con el uso general de los emojis en Twitter. Para esta confrontación, se ha recabado la lista de los 10 emojis más utilizados en Twitter un día y hora cualquiera a través de la página web Emojitracker, una herramienta que recoge el uso de los emojis en esa plataforma en tiempo real (ver Tabla 3 en la sección 4.2). Al no disponer de datos en tiempo real sobre el uso de los emojis el $8 \mathrm{M}$ y al observar que la variación por día en el uso de los emojis en el corpus es mínima, la comparación se ha basado en la lista de los 10 emojis más usados a lo largo de la semana de recogida de datos (Tabla 2, sección 4.2).

Para explorar el uso concreto de los pictogramas más frecuentes, se ha realizado un análisis cualitativo-discursivo de una muestra de alrededor del $1 \%$ del corpus. Con la ayuda de unos ejemplos representativos, se comenta en este trabajo el uso que se hizo de los emojis más frecuentes. Los resultados del análisis se detallan en la siguiente sección.

\section{Resultados}

\subsection{Tipos de emojis más utilizados en el corpus}

El corpus contiene un amplísimo abanico de emojis. Para ofrecer una primera aproximación a esta variedad de elementos gráficos, nos hemos centrado en primer lugar en los emojis más frecuentes. La herramienta Brandwatch extrae de forma automática los 300 emojis más utilizados en todo el corpus (es decir, los que están repetidos más de 50 veces), que reproducimos en la Figura 1 (a mayor tamaño, mayor uso).

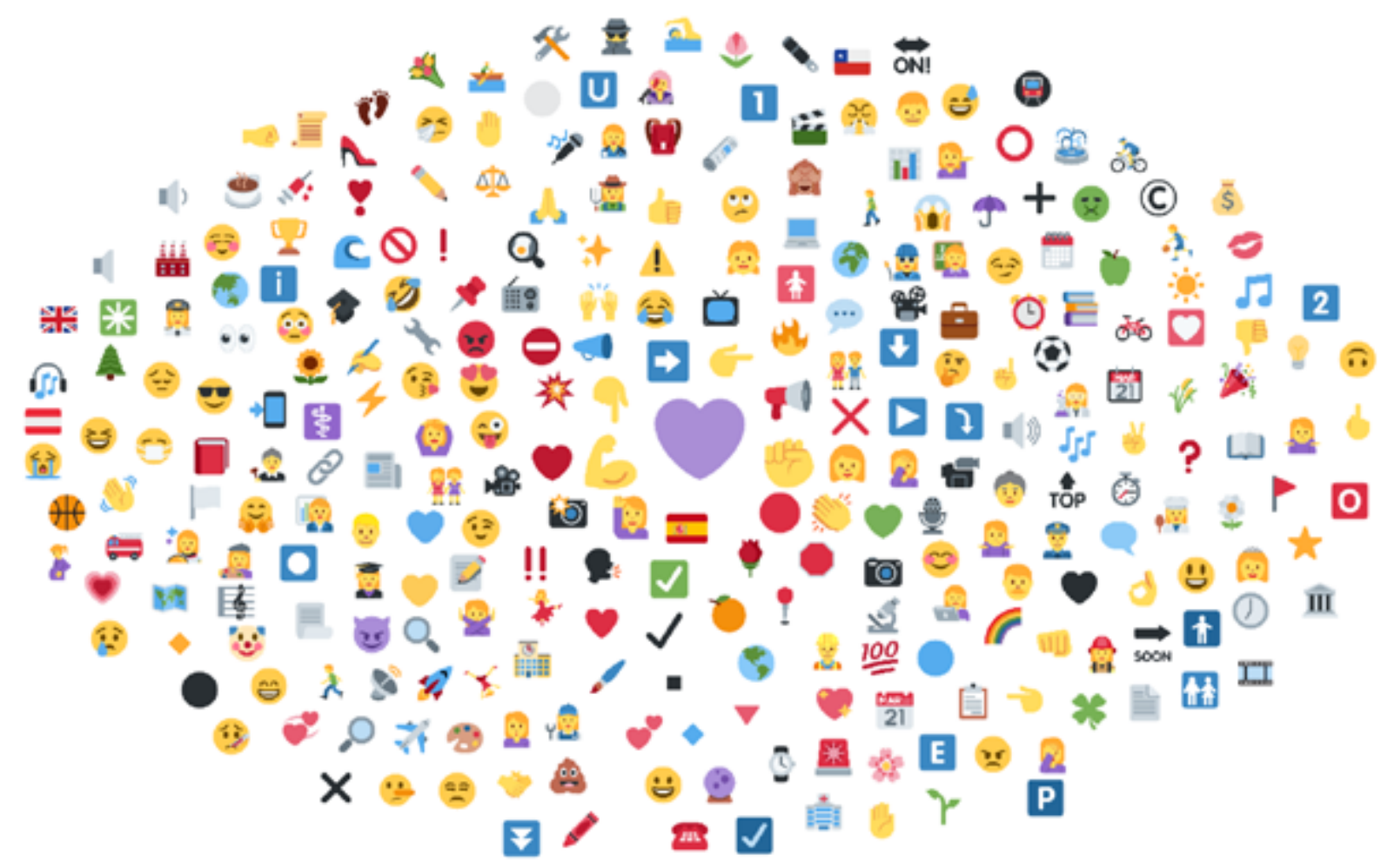

Figura 1: Los 300 emojis más utilizados 
Tomando como referencia las categorías empleadas por Emojipedia, vemos que a lo largo de la lista de los 300 emojis más utilizados aparecen todas las tipologías de emojis (Tabla 1).

Tabla 1. Categorías de emojis más utilizadas

\begin{tabular}{|l|c|}
\hline Categoría & N. emojis \\
\hline caras, personas y gestos & 109 \\
\hline animales y naturaleza & 26 \\
\hline comida y bebida & 5 \\
\hline actividades & 12 \\
\hline viajes y lugares & 12 \\
\hline objetos & 49 \\
\hline símbolos & 73 \\
\hline banderas & 14 \\
\hline
\end{tabular}

Como suele ser habitual incluso en otros contextos digitales, como los mensajes de la aplicación WhatsApp (Sampietro, 2016), la categoría más utilizada es "caras, personas y gestos", que incluye, entre otros, las reconocidas caritas amarillas, representaciones más o menos realistas de personas (realizando gestos, con diferentes atuendos, parejas y familias) y movimientos de las manos. Al ser la categoría más numerosa, no debería sorprender que el corpus incluya mucha variedad de pictogramas pertenecientes a esta tipología (109 emojis diferentes). Entre estos tipos de emojis, en el corpus se encuentran sobre todo varios tipos de gestos, algunas caritas amarillas, personas y parejas.

Resultan más sorprendentes la segunda y la tercera categoría más empleadas, símbolos (73 emojis) y objetos (49 emojis). Entre los primeros, el catálogo de Unicode incluye corazones de varios colores (negro, azul, amarillo), formas (un punto exclamativo en forma de corazón) y características (como el corazón que crece o el que es rodeado de estrellitas). Los otros emojis de esta categoría son diferentes tipos de símbolos, como flechas apuntando en varias direcciones, figuras geométricas (círculos, triángulos, etc.), señales variadas (prohibido el paso, alto voltaje, peligro...) y notas musicales, entre otros. Como se verá a continuación con más detalle, el emoji más utilizado en el corpus pertenece a esta categoría, pues es, y con mucha diferencia, el corazón de color violeta. Otros emojis recurrentes en el corpus que pertenecen a esta categoría son varios tipos de megáfonos y altavoces, flechas apuntando en varias direcciones y el símbolo de Venus.

En cuanto a los objetos, la lista de los emojis más empleados en el corpus incluye principalmente pictogramas relacionados con la comunicación, como cámaras de fotos, teléfonos, radio, televisión, antenas, y también libros, periódicos, libretas, etc. No se encuentran otras imágenes típicas de esta categoría, como armas, iconografía festiva, calendarios y material de oficina (excepto libros).

Los animales y los elementos naturales son la siguiente categoría (26 emojis diferentes), entre los que en el corpus destacan especialmente las representaciones del globo terráqueo, flores y otros vegetales; el único animal que se encuentra es un mono que se tapa los ojos. Menos frecuentes son las banderas (aparecen 14 banderas diferentes en 
la lista de los 300 emojis más usados, la más frecuente de las cuales es la española), las actividades (deportes, pintura, cine) y las imágenes relacionadas con los viajes (medios de transporte) y el entorno urbano (edificios). Solo 5 de los 300 emojis más frecuentes en el corpus representan comida y bebida.

Si nos centramos en los 10 emojis más utilizados en el corpus a lo largo del período analizado (Tabla 2), notamos que no aparece ninguna de las caritas amarillas que en otros contextos suelen encabezar la lista de emojis más frecuentes. Las únicas figuras antropomórficas son una persona con el brazo levantado (séptimo emoji más empleado) y una mujer (décimo emoji de la lista). Los demás son símbolos (corazón, flecha) y gestos (bíceps, puño, dedo índice).

Tabla 2. Los 10 emojis más utilizados en el corpus (4-9 de marzo de 2019) ${ }^{4}$

\begin{tabular}{|l|l|l|}
\hline N. Descripción oficial (traducción) & Apariciones \\
\hline 1 & purple heart (corazón violeta) & 47.697 \\
\hline 2 & flexed bíceps (bíceps) & 16.722 \\
\hline 3 & raised fist (puño) & 12.733 \\
\hline 4 & white down pointing backhand index (dedo indicando hacia abajo) & 9.220 \\
\hline 5 & public address loudspeaker (megáfono) Fl & 8.877 \\
\hline 6 & white right pointing backhand index (dedo indicando hacia la derecha) & 6.541 \\
\hline 7 & happy person raising one hand (persona feliz levantando un brazo) 6 & 6.326 \\
\hline 8 & black rightwards arrow (flecha hacia la derecha) $\rightarrow$ & 4.773 \\
\hline 9 & flag for Spain (bandera española) E & 4.354 \\
\hline 10 & woman (mujer) (6. & 3.790 \\
\hline
\end{tabular}

Tabla 3. Los emojis más utilizados en Twitter la mañana del día 29/11/2019

\begin{tabular}{|l|l|c|}
\hline N. & Descripción oficial (traducción) & Apariciones \\
\hline 1 & face with tears of joy (cara llorando de la risa) & 2.629 .715 .197 \\
\hline 2 & heavy black heart (corazón) & 1.266 .532 .132 \\
\hline 3 & black universal recycling symbol (símbolo de reciclaje) $\$$ & 965.309 .208 \\
\hline 4 & smiling face with heart-shaped eyes (cara con corazones en los ojos) & 950.715 .797 \\
\hline 5 & loudly crying face (cara llorando intensamente) & 797.572 .875 \\
\hline 6 & black heart suit (as de corazones) & 737.741 .081 \\
\hline 7 & smiling face with smiling eyes (cara sonriente con ojos sonrientes) & 624.978 .007 \\
\hline 8 & unamused face (cara sin diversión) & 497.130 .555 \\
\hline 9 & two hearts (dos corazones) & 488.478 .639 \\
\hline 10 & face throwing a kiss (cara que manda un beso) & 470.710 .078 \\
\hline
\end{tabular}

4. La herramienta no distingue entre colores de piel, por lo que el resultado que se indica es la totalidad de emojis que representan el gesto, independientemente de la tonalidad de piel escogida por el usuario. En la tabla se incluye la versión por defecto (de color amarillo). 
Si comparamos esta lista de los emojis más utilizados alrededor del $8 \mathrm{M}$ con el uso general de los emojis en Twitter en un instante cualquiera de un día normal, según datos de Emojitracker (Tabla 3), notamos varias diferencias.

En primer lugar, el emoji más frecuente en Twitter muy por encima de los demás suele ser face with tears of joy, la carita que se ríe hasta las lágrimas. En el corpus del 8M este emoji aparece en la posición 31 de la lista de los 300 emojis más utilizados. Otras caritas amarillas con expresiones positivas muy frecuentes en Twitter que no están entre los emojis más repetidos en el corpus analizado son face with heart-shaped eyes (una carita en las que los corazones sustituyen los ojos, cuarto emoji más frecuente en Twitter, $30^{\circ}$ en el corpus), smiling face with smiling eyes (una carita sonriente sonrojada, séptimo emoji más utilizado, $52^{\circ}$ en los tuits sobre el $8 \mathrm{M}$ ) y face throwing a kiss (la carita que manda un beso, décimo emoji más utilizado en Twitter, $53^{\circ}$ en el corpus estudiado), entre otros.

Aunque la lista de Emojitracker incluye varios tipos de corazones rojos, para encontrar el de color violeta hay que bajar respectivamente hasta el puesto 29 (purple heart). El bíceps (segundo emoji más utilizados en el corpus, como se analiza a continuación) se encuentra en el puesto 66 de la lista de Emojitracker. En general, estos datos indican que el uso de los emojis que se hizo alrededor del $8 \mathrm{M}$ es bastante diferente del uso general de los emojis en Twitter, dónde generalmente prevalecen las expresiones faciales positivas y los corazones rojos.

\subsection{Uso de los 10 emojis más frecuentes en el corpus}

Como se acaba de observar, el emoji que más se utiliza con diferencia en nuestro corpus es el corazón violeta (47.697 apariciones), muy por encima del bíceps, que es el segundo emoji más empleado (16.722). El corazón violeta se usa a veces como simple adición a un tuit que ya alude visual o textualmente al $8 \mathrm{M}$, como vemos en el tuit reproducido en la Figura 2.

Este \#8deMarzo ponemos en valor el feminismo, uno
de los movimientos sociales más importantes de los
últimos tiempos, cuya lucha ha sido imprescindible
para la conquistar de los avances alcanzados en
igualdad.
Por todo lo conquistado y por todo lo que queda por
conquistar

Figura 2: Tuit del 4 de marzo con corazón violeta ${ }^{5}$

A veces este emoji se usa incluso para contextualizar visualmente un tuit, de manera análoga a la función que a veces cumplen los hashtags, para que se entienda que su contenido, formulado de manera general, se refiere al 8M (Figura 3).

El segundo emoji más empleado es flexed biceps. Convencionalmente, contraer el bíceps es un gesto asociado con la idea de fuerza (Nakamura y Zeng-Treitler, 2012), puesto que es uno de los músculos más potentes de nuestro cuerpo. En la comunicación digital

5. En la reproducción de los tuits se ha eliminado (siempre que fuera posible) el nombre del usuario para poder preservar su privacidad y se ha omitido la reproducción de fotos y vídeos subidos por los usuarios, en los que se podían reconocer a manifestantes u otras personas. Si la autoría es pública y relevante (como, por ejemplo, si el tuit procede de un partido en concreto), se menciona directamente en el texto. 
está empezando a emplearse de forma casi convencional como manera de dar ánimos al interlocutor (Bennett, 2014). Como pocos mensajes en el corpus parecen respuestas directas a otros usuarios, es posible que el uso que se hace de este emoji en el corpus refleje más bien la idea de fuerza colectiva, aludiendo a que el movimiento es poderoso (Figura 4).

\section{Enamorada de esa mujer 93 años y luchadora hasta el final \#8M}

Figura 3: tuit del 9 de marzo con corazón violeta

\section{Manifestación \#8M \\ Jaén \#DiaDeLaMujer \\ Eran sólo unas "poquillas" \\ Vía WhatsApp, no sabemos 31 autor.}

Figura 4: tuit del 9 de marzo con emoji del bíceps flexionado

Otros dos pictogramas que representan manos o brazos están relacionados en cierta medida con la gestualidad típica de la protesta o de la resistencia, como levantar el puño o el brazo (Lang, 2019). Azhar (2017b) considera que el emoji del puño se está incluso convirtiendo en una manera convencional de marcar en Twitter las protestas de carácter progresistas, entre las que podemos incluir la del 8M (Figura 5).

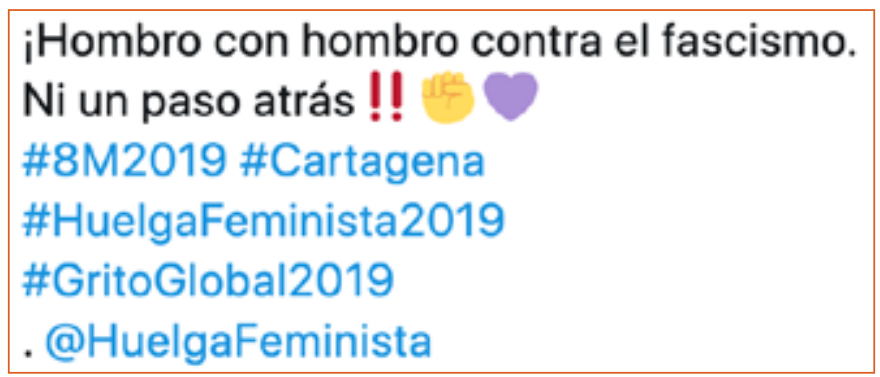

Figura 5. Tuit del 9 de marzo con emoji del puño en alto

Otros emojis tienen una función similar a los gestos que se utilizan en la comunicación cara a cara. Por ejemplo, el emoji del dedo índice señalando hacia a la derecha se puede considerar un gesto deíctico (Tomasello, 2013). Es más, parece incluso una reproducción moderna de la "manecilla", un signo utilizado antiguamente para señalar parte del texto (Luque Moreno, 2006). En la Figura 6 vemos que el dedo hacia abajo indica un tuit incrustado (que contiene una imagen de los políticos que por aquel entonces lideraban los partidos Vox, PP y Ciudadanos). En líneas generales, el dedo hacia abajo suele señalar tuits incrustados, vídeos o imágenes adjuntas (a veces incluso con una referencia deíctica como "aquí", "esto", etc.), mientras que los emojis que indican hacia la derecha y la flecha suelen preceder un enlace externo. 


\section{No es una huelga por la mujer sino por ideología política. Demostración}

Figura 6: tuit del 8 de marzo con emoji indicando hacia abajo

Destaca también el megáfono, que sirve como curiosa manera de expresar visualmente información de otros sentidos, en este caso, la audición. Aunque en la comunicación digital convencionalmente se señala un tono de voz más alto a través del uso de mayúsculas (Darics, 2010), en el corpus esta función es asumida por el megáfono (Figura 7). En la nube de los emojis más frecuentes que reproducimos en la Figura 1 también se encuentran otros tipos de altavoces y amplificadores, que se usan con la misma función.

\section{"Aquí está, aquí se ve, o feminismo galego en pé" I Así suena Vigo en la \#HuelgaFeminista2019}

Figura 7: tuit del 8 de marzo con emoji del megáfono

Los emojis antropomórficos también se encuentran en la lista de pictogramas más utilizados. El que representa una mujer con el brazo levantado suele tener usos bastantes heterogéneos. A veces se emplea a modo de saludo o para indicar que se acude a la manifestación (Figura 8) o bien, de forma menos frecuente, como gesto de protesta, de manera similar al emoji del puño levantado. Nótese que el tuit reproducido en la Figura 8 hace referencia al Partido Socialista. Se intuye no solo por la alusión directa (iAquí estamos las y los socialistas!), sino también por la variación de la etiqueta \#LaEspañaQueQuieres, uno de los lemas de la precampaña socialista para las elecciones del 28 de abril de 2019. En ocasión de ese mismo acontecimiento electoral, el PSOE utilizó profusamente en internet y en sus actos de campaña un corazón (generalmente rojo, o blanco sobre fondo rojo) precedido de una barra (/). En el tuit de la Figura 8 también se encuentra esa secuencia, una barra y un corazón, en este caso violeta, en coherencia con los colores del 8M.

\section{- 1 ¡Aquí estamos las y los socialistas! Como cada \#8M $\%$ apoyando la lucha de las mujeres. \\ La \#igualdad entre hombres y mujeres es el corazón de la democracia. \\ \#LaEspañaQueQuieresEsFeminista}

Figura 8: tuit del 8 de marzo con emoji de la persona con brazo levantado

El emoji que representa una mujer se usa para indicar gráficamente el hecho de que se trata del día de la mujer o en secuencia con otros emojis que representan mujeres de diferentes edades o con varios tipos de atuendos profesionales (Figura 9). 


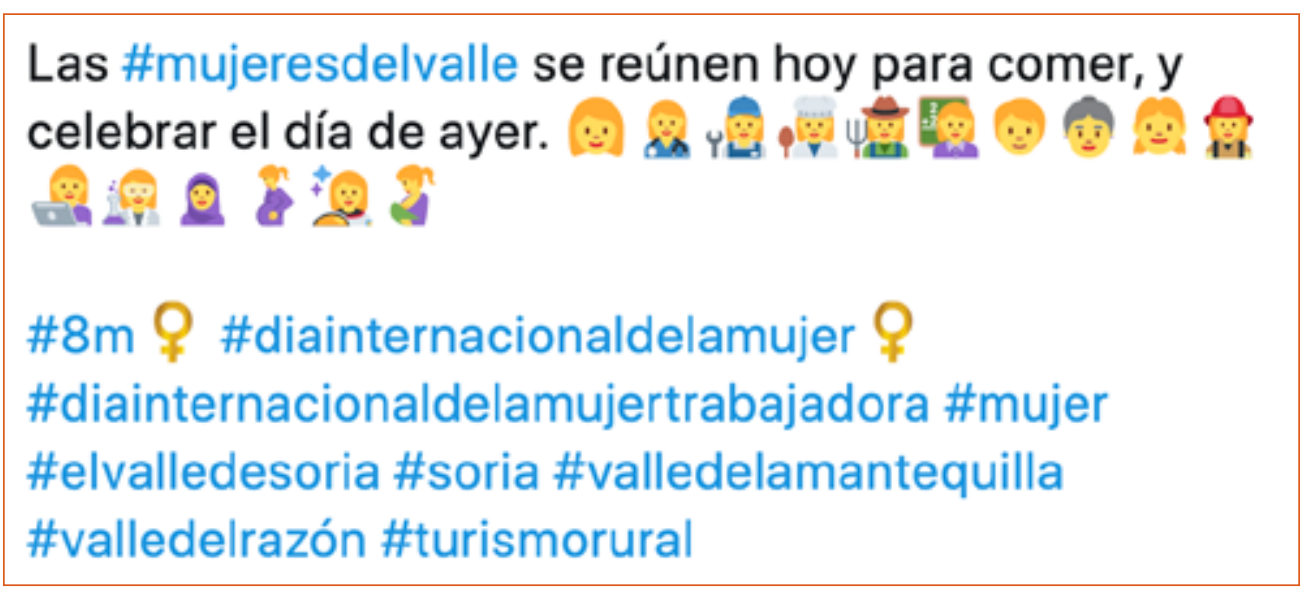

Figura 9: tuit del 9 de marzo con emojis que representan a mujeres

La única bandera presente en la lista de emojis más usados, como es de imaginar por las características del corpus, es la española (noveno emoji más frecuente). Además de hallarse junto con emojis de otras banderas nacionales en listas que presentan datos relevantes sobre la condición de la mujer en varios países, este emoji se encuentra a menudo en los tuits publicados por el partido Vox, contrario a la manifestación y a la huelga (Figura 10). Vox es un partido español de derecha radical, fundado en 2013 por ex dirigentes y militantes del Partido Popular. Entre los rasgos del partido, Ferreira (2019) reconoce que en la ideología de Vox es central el nacionalismo, con incluso tintes xenófobos. Como indica explícitamente este autor (Ferreira, 2019, p. 86): “Un desacomplejado nacionalismo español vertebra y está constantemente presente en el discurso, las propuestas y la escenografía de Vox". Con respecto a este último punto, destaca la asidua presencia de la bandera española en los actos y mítines del partido. En el ámbito digital, esto se traduce en el uso frecuente del emoji de la bandera española (Sampietro y Sánchez Castillo, 2020). Para ratificar esta conexión entre la bandera y el partido, se observa que en los tuits de nuestro corpus que contienen la bandera española, también se pueden encontrar algunas etiquetas difundidas por esa formación política, como \#EspañaViva, que se refiere a los seguidores de Vox, o \#NoHablesEnMiNombre, un hashtag difundido por las mujeres afines a Vox para distanciarse de las reivindicaciones del 8M.

\section{7 \#NoHablesEnMiNombre "Yo también soy mujer y quiero los mismos derechos para mi hija y para mi hijo". \\ X Las mujeres de la \#EspañaViva 2 rompen con la huelga de las feministas supremacistas}

Figura 10: tuit del 7 de marzo con bandera española

\section{Discusión}

Internet y las redes sociales se han posicionado en los últimos tiempos como herramientas para la organización de las protestas y la difusión de lemas que abordan el significado cultural y político de las reivindicaciones feministas (Chenou y Cepeda Másmela, 2019; Pavan y Mainardi, 2018). Este trabajo ha analizado otro elemento simbólico bastante inexplorado relacionado con esas reivindicaciones, el uso de los emojis en Twitter en ocasión de las exitosas manifestaciones del 8M de 2019. 
En respuesta a la primera pregunta de investigación, los emojis más utilizados en el corpus son los de la categoría "caras, personas y gestos" $y$, en segundo lugar, los que indican símbolos, como los corazones. El corazón violeta, de hecho, es con diferencia el emoji más empleado en el corpus. Azhar (2017a) observó que este emoji también era de los más utilizados en los tuits categorizados con la etiqueta \#MeToo. Este color está tradicionalmente asociado con la mujer. El movimiento International Women's Day, de hecho, refiere en su página web que ha adoptado el color violeta porque al parecer fue uno de los que se utilizaron en la primera marcha de mujeres para mejorar sus condiciones laborales en marzo de 1908 (International Women's Day, n.d.).

Los emojis de la categoría "caras, personas y gestos" más frecuentes en el corpus son diferentes tipos de gestos, entre los que destaca el tercer pictograma más usado, el del puño en alto. Como observa Azhar (2017b), este emoji se está convirtiendo en una manera convencional de simbolizar las protestas, y su uso frecuente en nuestro corpus (en especial los días 8 y 9 de marzo) ratifica esta observación. Los emojis del puño y del bíceps también se encuentran en la lista de pictogramas más utilizados en el corpus sobre el movimiento \#MeToo (Azhar, 2017a), aunque se usan en menor medida. Una de las razones podría ser que, pese a que también sean gestos relacionados con la fuerza y la rebelión ante la injusticia, el corpus recogido por Azhar (2017a) no coincidió concretamente con una protesta o manifestación, sino más en general con una muestra de activismo digital.

Sería interesante analizar si en otras situaciones de protesta relacionadas con la cuestión feminista (por ejemplo, el Día Internacional contra la Violencia de Género o concentraciones convocadas en protesta contra delitos sexuales o feminicidios) también se emplea este emoji. También se podría valorar si hay diferencias culturales en su uso, ya que, en otros países, por motivos sociales o culturales, podrían utilizarse otros colores. Por ejemplo, las protestas en favor de la legalización del aborto en Argentina, otra importante reivindicación feminista, se relacionaron con el color verde (Cantamutto y Vela Delfa, este volumen). Se podría investigar el uso de corazones verde y violeta en Twitter en las movilizaciones de carácter feminista en ese país.

En respuesta a la segunda pregunta de investigación, el análisis ha mostrado que el uso de los emojis en tuits que contienen una serie de etiquetas y palabras relacionadas con el evento en cuestión es diferente del uso de los emojis en general en esa red social. Aunque la categoría de pictogramas más frecuente es la que incluye caras, representaciones de personas y gestos, como en el uso general, un análisis pormenorizado de los emojis más utilizados en el corpus muestra claras diferencias con el uso general en Twitter un día cualquiera. Si las etiquetas sirven para articular un flujo de mensajes en torno a lemas y discursos concretos, el uso de pictogramas por parte del activismo revela la manera en la que estos elementos pueden contribuir a expresar la emoción y simbología propias del movimiento feminista.

En primer lugar, las caritas amarillas, que dominan la lista de emojis más frecuentes en Twitter en general, no se encuentran en la lista de los 10 emojis más utilizados en nuestro corpus. Comparando con el uso de los emojis en Twitter un día cualquiera, en nuestro corpus hay que bajar hasta el puesto 31 para encontrar el emoji más empleado en Twitter, face with tears of joy o hasta el 53 para hallar el más utilizado en WhatsApp, la carita que manda el beso (Sampietro, 2016). Un corazón es el segundo emoji más utilizado en Twitter en general, pero hay una diferencia clave con el corazón empleado en nuestro corpus: en Twitter prevalece el corazón rojo, que se usa para indicar de 
forma más genérica afecto, apreciación, amor o para reproducir el comando "me gusta" de esa misma plataforma, mientras que el corpus del $8 \mathrm{M}$ incluye una gran cantidad de corazones de color violeta, que, como hemos señalado, se refieren explícitamente al feminismo y al Día Internacional de la Mujer.

Estos hechos sugieren que las personas que participaron en Twitter con motivo del 8 de marzo, utilizaron un tono serio y reivindicativo, y su participación es bastante diferente del tono general que se encuentra en esa red social, caracterizado por cierta alegría y expresión de afecto. Estas diferencias también se expresan en los gestos: en la lista de emojis del 8M no encontramos reproducciones como el OK o la V de victoria, que son muy frecuentes en Twitter. El 8M prevalecieron los bíceps (para mostrar fuerza), el puño en alto (símbolo de protesta y resistencia), además de gestos deícticos que señalan gráficamente información adicional. También están relacionados con la difusión de información los emojis relativos a la iconografía de la comunicación, como el megáfono, la radio, la cámara de fotos, etc., cuyo uso no es tan frecuente en esa red social en general.

De hecho, en referencia a la tercera pregunta de investigación, el corpus muestra ciertos patrones de uso específicos. El corazón violeta sirve generalmente para contextualizar un tuit, el bíceps y el puño para mostrar fuerza o participación, los dedos o flechas indican enlaces o contenido adicional, las mujeres ilustran las protagonistas de la efeméride. Los pictogramas se adaptan a las tipologías de mensajes correspondientes a momentos específicos de la protesta, que se desplazan de la información sobre esta hasta la llamada a la (des)movilización, la retransmisión en directo y los mensajes de apoyo.

Se pueden también vislumbrar algunas diferencias en el uso de los emojis según la ideología política. El partido de derecha Vox, por lo menos en el corpus que se analiza, parece haberse apropiado del emoji que representa la bandera española, en coherencia con la profusión de símbolos nacionalistas que caracteriza la ideología y la escenografía del partido (Ferreira, 2019). La participación de este partido en las movilizaciones se puede vincular al resurgimiento de movimientos fundamentalmente machistas que se posicionan contra los postulados de las protestas del 8M (Banet-Weiser y Miltner, 2016). Podría ser interesante analizar en un futuro si en otras situaciones, como, por ejemplo, coincidiendo con la campaña electoral para las Elecciones Generales, el uso del emoji de la bandera también es prerrogativa del partido Vox o si su uso se reparte entre los partidos que tienen implicación en la política nacional. De otra manera: el emoji de la bandera de España facilitaría reconocer la apropiación de esta fórmula de identidad nacional con el ideario de partidos políticos específicos.

También hemos observado que el corazón violeta en los tuits del PSOE puede ir precedido de una barra (/), que parece recordar la simbología visual que dominó la campaña electoral del partido en ocasión de las Elecciones Generales del 28 de abril de 2019. Otros trabajos podrían analizar si, coincidiendo con otros tipos de eventos en los que hay una mayor confrontación entre partidos, como una campaña electoral, el uso de corazones de varios colores en Twitter varía más coincidiendo con los colores de cada partido. En el caso de España, por ejemplo, podría usarse más el rojo en los tuits del PSOE, el violeta en los de Unidas Podemos, el verde para los de Vox, el azul en los del Partido Popular y el naranja en las publicaciones de Ciudadanos. Si los usuarios se apropiaran de estos usos partidistas de los emojis, se mostraría la capacidad de los agentes institucionales para intervenir en el debate público y plantear visualmente sus posicionamientos ideológicos concretos. 


\section{Conclusiones}

El presente artículo es uno de los primeros trabajos sistemáticos que analizan el uso de los emojis en Twitter coincidiendo con eventos relevantes, en este caso las manifestaciones y paros del $8 \mathrm{M}$ de 2019. El análisis ha señalado que su uso es diferente del que generalmente se encuentra en la red social, ya que prevalece un uso simbólico de estos pictogramas para mostrar de forma solidaria participación en el evento, contextualizar el tuit, señalar el éxito de la manifestación y enfatizar rasgos visuales o incluso fónicos del mensaje. Se ha mostrado que el uso de los emojis varía dependiendo del contenido del tuit e incluso, hasta cierta medida, de la ideología política. Algunos emojis se utilizan mayormente de forma icónica, como los que se emplean para señalar enlaces, mientras que otros, como el corazón violeta, el puño en alto y el bíceps, se utilizaron de forma más simbólica acompañando a los tuits más afines a la protesta. Coincidimos con Azhar (2017b) en identificar el emoji del puño en alto como símbolo de la protesta progresista y nos parece de gran interés analizar la evolución en el uso de ese emoji en otras situaciones similares.

En posibles trabajos futuros, como se ha señalado en la discusión, podría ser interesante analizar el uso de los emojis coincidiendo con otros eventos importantes a nivel social y político, como otras huelgas o protestas, o incluso durante las campañas electorales. También podría ser muy enriquecedor analizar el uso de los emojis en otras redes sociales en las que estos pictogramas son frecuentes, como Instagram, que se está utilizando cada vez más como instrumento de comunicación política (Sampietro y Sánchez Castillo, 2020) y activismo (Tarullo y García, este volumen).

En conclusión, el trabajo muestra que los emojis no son solo simples toques de color que se añaden a los mensajes en redes sociales, sino que también se utilizan, a la par de eslóganes y lemas, como instrumento de expresión común en situaciones de protesta. La diferenciación de estos elementos gráficos en relación con su uso habitual demuestra su capacidad de apropiación por parte del movimiento feminista, que los emplea para la difusión de su discurso y la organización de sus movilizaciones. El uso del corazón violeta se está convirtiendo, por lo menos en España, en un verdadero símbolo del activismo feminista en Twitter.

\section{Referencias}

Al Rashdi, F. (2018): "Functions of emojis in WhatsApp interaction among Omanis", en Discourse, Context \& Media, vol. 26, pp. 117-126. doi: 10.1016/j.dcm.2018.07.001.

Augenbraun, E. (2011): "Occupy Wall Street and the limits of spontaneous street protest", en The Guardian (29 de septiembre). Disponible en: https://www.theguardian.com/ commentisfree/cifamerica/2011/sep/29/occupy-wall-street-protest [Consulta 2 de diciembre de 2019].

Azhar, H. (2016a): "The Emojis of Great Brexit", en Medium (1 julio). Disponible en: https://medium.com/prismoji/the-emojis-of-great-brexit-db53004c290e [Consulta 2 de diciembre de 2019]

Azhar, H. (2016b): “The top emojis of Election Day 2016", en Prismoji (26 de noviembre). Disponible en:https://prismoji.com/2016/11/26/the-top-emojis-of-election-day-2016/ [Consulta 6 de noviembre de 2019].

Azhar, H. (2017a): "Emojis of \#MeToo", en Prismoji (20 de octubre). Disponible en: https:// prismoji.com/2017/10/20/emojis-of-me-too/ [Consulta: 6 de noviembre de 2019]. 
Azhar, H. (2017b): "The Resistance Will Be Emojified", en Emojipedia (6 de febrero). Disponible en: https://blog.emojipedia.org/the-resistance-will-be-emojified/ [Consulta: 2 de diciembre de 2019].

Azuma, J. y Ebner, M. (2008): "A stylistic analysis of graphic emoticons: Can they be candidates for a universal visual language of the future?". En: Proceedings of the World Conference on Educational Media, Hypermedia and Telecommunications (ED-Media). Viena: Association for the Advancement of Computing in Education (AACE), pp. 972977.

Banet-Weiser, S. y Miltner, K.M. (2016). "\#MasculinitySoFragile: Culture, structure, and networked misogyny", en Feminist Media Studies, vol. 16, $\mathrm{n}^{\circ}$ 1, pp. 171-174. doi: 10.1080/14680777.2016.1120490.

Barbieri, F., Ballesteros, M. y Saggion, H. (2017): "Are Emojis Predictable?". En: Proceedings of the 15th Conference of the European Chapter of the Association for Computational Linguistics, vol. 2, pp. 105-111. Disponible en: https://www.aclweb.org/anthology/E172017.pdf

Barbieri, F., Espinosa-Anke, L. y Saggion, H. (2016): “Revealing patterns of twitter emoji usage in Barcelona and Madrid", en Frontiers in Artificial Intelligence and Applications, 288, pp. 239-244. doi: 10.3233/978-1-61499-696-5-239.

Bennett, J. (2014): "The Emoji Have Won the Battle of Words", en The New York Times (25 de julio). Disponible en: https://www.nytimes.com/2014/07/27/fashion/emoji-havewon-the-battle-of-words.html [Consulta: 2 de diciembre de 2019].

"Brandwatch" (2019): Wikipedia, la enciclopedia libre. Disponible en: https://en.wikipedia. org/wiki/Brandwatch [Consulta: 28 de noviembre de 2019].

Burge, J. (2017): “5 Billion Emojis Sent Daily on Messenger", en Emojipedia (17 de julio). Disponible en: https://blog.emojipedia.org/5-billion-emojis-sent-daily-on-messenger/ [Consulta: 28 de noviembre de 2019].

Burge, J. (2019): "Correcting the record on the first emoji set", en Emojipedia (8 marzo). Disponible en: https://blog.emojipedia.org/correcting-the-record-on-the-first-emojiset/ [Consulta: 28 de noviembre de 2019].

Campillo, I. (2019): "If we stop, the world stops': the 2018 feminist strike in Spain", en Social Movement Studies, vol. 18, $n^{\circ}$ 2, pp. 252-258. doi: 10.1080/14742837.2018.1556092

Cantamutto, L. y Vela Delfa, C. (2019): “Emojis frecuentes en las interacciones por whatsapp", en Círculo de Lingüística Aplicada a la Comunicación, Vol. 77, pp. 171-186. doi: 10.5209/CLAC.63282.

Cantamutto, L. y Vela Delfa, C. (este volumen): “¿De qué color es tu corazón? El uso de emojis en los procesos de activismo social", en Dígitos. Revista de comunicación digital, $\mathrm{n}^{\circ} 6$.

Casado-Molina, A.M., Rojas-de Gracia, M.M., Alarcón-Urbistondo, P.y Romero-Charneco, M (2019): "Exploring the Opportunities of the Emojis in Brand Communication: The Case of the Beer Industry", en International Journal of Business Communication, pp. 1-19. doi: $10.1177 / 2329488419832964$.

Chenou, J. M. y Cepeda-Másmela, C. (2019): “\#NiUnaMenos: Data Activism from the Global South", en Television and New Media, vol. 20, $\mathrm{n}^{\circ}$ 4, pp. 396-411. doi: 10.1177/1527476419828995.

Comisión 8M (2019): “Hacia la Huelga Feminista". Recuperado de: http:// 
hacialahuelgafeminista.org [Consulta: 13 de noviembre de 2019].

Danesi, M. (2016): The Semiotics of Emoji: The Rise of Visual Language in the Age of the Internet. London: Bloomsbury.

Darics, E. (2010): "Politeness in computer-mediated discourse of a virtual team", en Journal of Politeness Research. Language, Behaviour, Culture, vol. 6, n 1, pp. 129-150. doi: 10.1515/jplr.2010.007.

Eslen-Ziya, H. (2013): "Social media and Turkish feminism: New resources for social activism", en Feminist Media Studies, vol. 13, no 15, pp. 860-870. doi: 10.1080/14680777.2013.838369.

"Etiqueta (internet)" (2019): Wikipedia, la enciclopedia libre. Recuperado de: https:// es.wikipedia.org/wiki/Etiqueta_(internet) [Consulta: 6 de noviembre de 2019].

Fernández Romero, D., y Sánchez Duarte, J. M. (2019): “Alianzas y resistencias feministas en Facebook para la convocatoria del 8M en España", en Convergencia. Revista de Ciencias Sociales, vol. 81. doi: 10.29101/crcs.v26i81.11943

Ferreira, C. (2019): "Vox como representante de la derecha radical en España: un estudio sobre su ideología", en Revista Española de Ciencia Política, n 51, pp. 73-98. doi: 10.21308/recp.51.03.

Galdón, C. (2018): “Cosmovisiones feministas en clave generacional. Del movimiento $15 \mathrm{M}$ a la Huelga Feminista del 8M", en Encrucijadas - Revista Crítica de Ciencias Sociales, vol. 16, v1602. Recuperado de: http://www.encrucijadas.org/index.php/ojs/article/ view/411/312 [Consulta 2 de diciembre de 2019].

Ge, J. y Gretzel, U. (2018): "Emoji rhetoric: a social media influencer perspective", en Journal of Marketing Management, vol. 34, $\mathrm{n}^{\circ}$ 15-16, pp. 1272-1295. doi: 10.1080/0267257X.2018.1483960.

Ge, J. y Herring, S. C. (2018): "Communicative functions of emoji sequences on Sina Weibo", en First Monday, vol. 23, n 11. doi: 10.5210/fm.v23i11.9413.

Goméz, M.V.(2019). “Una movilización masiva exhibeen las calles la fuerza del feminismo", en El País (9 de marzo). Disponible en: https://elpais.com/sociedad/2019/03/08/ actualidad/1552079524_186232.html [Consulta 8 de noviembre de 2019].

Herring, S. C. y Dainas, A. (2017): 'Nice Picture Comment!' Graphicons in Facebook Comment Threads". En: Proceedings of the Fifthieth Hawaii International Conference on System Sciences, pp. 2185-2194. Recuperado de: https://www.semanticscholar. org/paper/\%22Nice-Picture-Comment!\%22-Graphicons-in-Facebook-Herring-Dainas/ e920f862cfa075aead9f7f7c15632599ec17e534

Hutchby, I. (2001): "Technologies, Texts and Affordances", Sociology, vol. 35, n² 2, pp. 441-456.

International Women's Day (n.d.): "About International Women's Day". Disponible en: https://www.internationalwomensday.com/About [Consulta: 7 de noviembre de 2019].

Jaeger, S. R., Cardello, A. V. y Schutz, H. G. (2013): “Emotion questionnaires: A consumercentric perspective", en Food Quality and Preference, Vol. 30, n 2, pp. 229-241. doi: 10.1016/J.FOODQUAL.2013.05.015.

Jaeger, S. R., Roigard, C. M. y Ares, G. (2018): “Measuring consumers' product associations with emoji and emotion word questionnaires: case studies with tasted foods and written stimuli", en Food Research International, Vol. 111, pp. 732-747. doi: 10.1016/J. 
FOODRES.2018.04.010.

Kabir, H. and Marlow, D. W. (2019): "Emojis in Textual-Based Communication Among College Students: A Study in Perception and Frequency". En: Meiselwitz, G. (ed.): Social Computing and Social Media. Design, Human Behavior and Analytics. HCII 2019. Lecture Notes in Computer Science. Springer Nature Switzerland, pp. 336-344. doi: 10.1007/978-3-030-21902-4_24.

Kmieckowiak, T. (2017): "Emojis Lead up to 47.7\% More Interactions on Instagram", en quintly. Disponible en: https://www.quintly.com/blog/instagram-emoji-study [Consulta: 5 de diciembre de 2019].

Lang, H. (2019): “\#MeToo: A Case Study in Re-Embodying Information", en Computers and Composition, vol. 53, pp. 9-20. doi: 10.1016/j.compcom.2019.05.001.

Luque Moreno, J. (2006): Puntos y comas. La grafía del articulación del habla. Granada: Editorial Universidad de Granada.

Matamoros-Fernández, A. (2018): "Inciting anger through Facebook reactions in Belgium: The use of emoji and related vernacular expressions in racist discourse", en First Monday, vol. 23, n 3. Disponible en: https://firstmonday.org/ojs/index.php/fm/ article/view/9405/7571 [Consulta: 2 de diciembre de 2019].

Miyake, K. (2007): “How young Japanese express their emotions visually in mobile phone messages: A sociolinguistic analysis", en Japanese Studies, vol. 27, n 1, pp. 53-72. doi: 10.1080/10371390701268646.

Nakamura, C. y Zeng-Treitler, Q. (2012): “A taxonomy of representation strategies in iconic communication", en International Journal of Human-Computer Studies, vol. 70, $\mathrm{n}^{\circ}$ 8, pp. 535-551. doi: 10.1016/j.ijhcs.2012.02.009.

Núñez Puente, S., D’Antonio Maceiras, S. y Fernández Romero, D. (2019): “Twitter activism and ethical witnessing: Possibilities and challenges of feminist politics against gender-based violence", en Social Science Computer Review, 1-17. doi: 10.1177/0894439319864898

Núñez Puente, S., y Fernandez Romero, D. (2017): “Narrativas transformadoras y testimonio ético: las estrategias discursivas de la Plataforma Feminista 7N, Contra las Violencias Machistas", en Index Comunicación, Vol. 7, n³, pp. 269-281. Recuperado de http://journals.sfu.ca/indexcomunicacion/index.php/indexcomunicacion/article/ view/341/331.

Orgad, S., y Gill, R. (2019): "Safety valves for mediated female rage in the \#MeToo era”, en Feminist Media Studies, vol. 19, n4, pp. 596-603. doi: 10.1080/14680777.2019.1609198.

Pavan, E., y Mainardi, A. (2018): "Striking, marching, tweeting: Studying how online networks change together with movements", en Partecipazione e Conflitto, Vol. 11, $\mathrm{n}^{\circ}$ 2, pp. 394-422. doi: 10.1285/i20356609v11i2p394.

Parkwell, C. (2019): “Emoji as social semiotic resources for meaning-making in discourse: Mapping the functions of the toilet emoji in Cher's tweets about Donald Trump", en Discourse, Context \& Media, Vol. 30, p. 100307. doi: 10.1016/J.DCM.2019.100307.

Pérez-Sabater, C. (2019): "Emoticons in Relational Writing Practices on WhatsApp: Some Reflections on Gender". En: Bou-Franch, P. y Garcés-Conejos Blitvich, P. (eds.), Analyzing Digital Discourse: New Insights and Future Directions. Cham: Springer, pp. 163189. doi: 10.1007/978-3-319-92663-6_6.

Riordan, M. A. (2017): “Emojis as tools for emotion work: Communicating affect in text 
messages", en Journal of Language and Social Psychology, Vol. 36, n 5, pp. 549-567. doi: 10.1177/0261927X17704238.

Ruiz Franco, R. (2018): "El valor histórico de la celebración del 8 de marzo como Día Internacional de las Mujeres", en Femeris, vol. 3, n 3, pp. 5.7. doi: 10.20318/ femeris.2018.4329

Sampietro (2016): Emoticonos y emojis. Análisis de su historia, difusión y uso en la comunicación digital actual. Tesis doctoral no publicada, Universitat de València.

Sampietro, A. (2019): “Cómo hacer palabras con emojis: sustitución y enfatización visual de vocablos en WhatsApp", en Revista de Estudios sobre el Discurso Digital, $\mathrm{n}^{\circ} 2$, pp. 1-33. doi: 10.24197/redd.2.2019.1-33

Sampietro, A. y Sánchez-Castillo, S. (2020): “La promoción de la imagen política en Instagram: Un estudio del perfil personal de Santiago Abascal (Vox) en 2018 / Building a political image on Instagram: A study of the personal profile of Santiago Abascal (Vox) in 2018", en Communication \& Society, vol. 33, n 1, pp. 169-184. doi: https://doi. org/10.15581/003.33.1.169-184.

Soranaka, K. y Matsushita, M. (2012): "Relationship between emotional words and emoticons in tweets". En: Proceedings of the 2012 Conference on Technologies and Applications of Artificial Intelligence. leee, pp. 262-265. doi: 10.1109/TAAI.2012.30.

Statista (2020): "Number of Twitter users worldwide from 2014 to 2020 (in millions)". Recuperado de: https://www.statista.com/statistics/303681/twitter-users-worldwide/ [Consulta: 14 de febrero de 2020].

Tang Khe Foon Hew, Y. (2019): "Emoticon, emoji, and sticker use in computer-mediated communication: A review of theories and research findings", en International Journal of Communication, vol. 13, pp. 2457-2483.

Tarullo, R. y García, M. (2020): “Hashtivismo feminista en Instagram: \#NiñasNoMadres de @actrices.argentinas", en Dígitos. Revista de comunicación digital, nº 6, pp. 31-54.

Tomasello, M. (2013): Los orígenes de la comunicación humana. Buenos Aires: Katz.

Trending topics del viernes 08 de marzo en España (n.d.): Trendinalia. Disponible en: https://www.trendinalia.com/twitter-trending-topics/spain/spain-190308.html [Consulta 8 de diciembre 2019].

"Twitter" (2020): Wikipedia, la enciclopedia libre. Recuperado de: https://en.wikipedia. org/wiki/Twitter [Consulta: 14 de febrero de 2020].

Wijeratne, S., Balasuriya, L., Sheth, A. y Doran, D. (2017): “A semantics-based measure of emoji similarity". En: Proceedings of the International Conference on Web Intelligence - WI '17, pp. 646-653 doi: 10.1145/3106426.3106490. 
158 